ROTUR, Revista de Ocio y Turismo

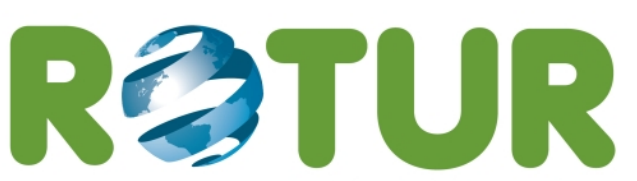

Vol. 14(1) (2020) pp. 81-93. ISSN-e 2695-6357

DOI: https://doi.org/10.17979/rotur.2020.14.1.5945

\title{
(cc) BY-SA
}

Nuevas tecnologías y difusión del turismo cultural: descubriendo a Goya con realidad aumentada

New technologies and cultural tourism: Discovering Goya with augmented reality

$\mathrm{M}^{\mathrm{a}}$ Sagrario Bernad Conde

Universidad de Zaragoza

sagrabernadc@gmail.com

https://orcid.org/0000-0001-6131-1011

Recibido/Received: 07-01-2020

Aceptado/Accepted: 31-01-2020

\section{RESUMEN}

La introducción de la realidad aumentada permite agregar información de interés para un destino turístico. Además, contribuye a aumentar la atracción e incrementar el mercado de visitantes. En la actualidad la adopción de tecnologias de realidad virtual aplicadas al patrimonio cultural está en un estadio de desarrollo. En este artículo se analizan esas tecnologias y, en particular, la app de Fuendetodos. Los resultados concluyen que el uso de la app permite aumentar la información y la experiencia vivida del visitante sobre Goya y la localidad de Fuendetodos.

Palabras clave: educación patrimonial; aprendizaje móvil; apps; realidad aumentada; Fuendetodos

\section{ABSTRACT}

Augmented reality is an effective tool for adding information and meaning to a tourist destination in order to increase its attractivenessand expand the site's potential tourist market. The use of virtual reality technology in the cultural heritage sector is still in the early stages. This article examines the adoption of these technologies for the promotion of cultural heritage, particularly in relation to the Fuendetodos tourist application. The results show that the use of this app increases the information available to users and enhances visitors' individual experience of Goya and the village of Fuendetodos.

Keywords: heritage education; mobile learning; apps; augmented reality; Fuendetodos

\section{CÓMO CITAR ESTE ARTÍCULO/ HOW TO CITE THIS ARTICLE}

Bernad Conde, María Sagrario (2020). Nuevas tecnologías y difusión del turismo cultural: descubriendo a Goya con realidad aumentada. Rotur, Revista de Ocio y Turismo, 14(1): 81-93 https://doi.org/10.17979/rotur.2020.14.1.5945 


\section{INTRODUCCIÓN}

El presente estudio se integra dentro de una línea de investigación compleja que pretende analizar el impacto de las nuevas tecnologías en el ámbito patrimonial. Con la implementación de la tecnología, el patrimonio cultural permite dar a conocer un territorio y soporta la oferta cultural de un destino. El desarrollo tecnológico y los dispositivos móviles han incrementado no sólo las herramientas a disposición de la educación, sino que se han transformado las estructuras de aprendizaje más tradicionales, generando la necesidad de integrarlos en el aprendizaje formal e informal. Partiendo de esta premisa, la aplicación de las nuevas tecnologías al ámbito de la educación patrimonial es el punto de partida de este trabajo. Su finalidad se centra en valorar cómo puede modificarse la percepción que los usuarios tienen de la aplicación de Fuendetodos para interpretar los itinerarios antes y después de la realización de las rutas propuestas con la ruta guiada de la aplicación de la localidad natal del pintor y genio universal Francisco de Goya y Lucientes, Fuendetodos. Tras el recorrido por las rutas propuestas se pretende responder a cuestiones como si el uso de nuevas tecnologías y en concreto la Realidad Aumentada influye en el grado de conocimiento que adquiere el usuario de la app del patrimonio de la localidad, si ha variado la percepción y conocimiento que tiene de la figura de Goya y conocer las limitaciones que presenta.

La utilización de la Realidad Aumentada ha permitido acercar un tipo de virtualidad más inmersiva y efectiva a todo tipo de usuarios (Fombona, Pascual y Madeira, 2012), generando interesantes estudios sobre el uso de la Realidad Aumentada en educación, aunque menor que en otras áreas (Cabero y Barroso 2016; Prendes, 2015). Los dispositivos de realidad aumentada son un nuevo medio de comunicación con experiencias inmersivas únicas y permiten insertar objetos o espacios virtuales en un escenario real.

La app objeto de análisis en esta investigación, Fuendetodos, cuenta con el apoyo económico del consorcio Goya-Fuendetodos de la Diputación de Zaragoza, es gratuita y solo puede activarse una vez que el visitante se encuentra en Fuendetodos. Incorpora la realidad aumentada, la geolocalización y la gamificación, el uso de los juegos como herramienta educativa.

El estudio se ha estructurado en cinco capítulos. Un primer apartado introductorio donde se contextualiza la investigación. Un segundo epígrafe se corresponde con el estado de la cuestión sobre la materia tratada, realizando una revisión bibliográfica del enfoque teórico del trabajo: la realidad aumentada y el turismo cultural. En el tercer apartado se plantean las hipótesis de partida, los objetivos y la metodologia utilizada en la investigación. Posteriormente, en el apartado cuarto se analizan los datos obtenidos tras el análisis de la app y se exponen los resultados más representativos. El último epígrafe recoge las conclusiones y, finalmente, se incluye la bibliografia.

\section{REALIDAD AUMENTADA Y PATRIMONIO CULTURAL}

Las TIC se han convertido actualmente en una herramienta para la difusión del patrimonio cultural. La UNESCO señala que el patrimonio es importante para la cultura y constituye el capital cultural de las sociedades contemporáneas. Contribuye a la revalorización continua de las culturas y de las identidades, y es un vehículo importante para la transmisión de experiencias, aptitudes y conocimientos entre las generaciones. Además, es fuente de inspiración para la creatividad y la innovación, que generan los productos culturales contemporáneos y futuros. El patrimonio cultural permite el acceso a la diversidad cultural y 
su disfrute: "Una ciudad inteligente y sostenible utiliza tecnologias de la información y la comunicación (TIC) para proporcionar una mejor calidad de vida a sus ciudadanos, la mejora de la eficiencia de los servicios y el desarrollo sostenible. Esta ciudad atiende a las necesidades de hoy $\sin$ sacrificar las necesidades de las generaciones futuras en lo que respecta a los aspectos económicos, sociales y ambientales" (UNESCO, 2014).

El estudio de Acción Cultural Española (ACE), en su anuario ACE 2017, analiza el impacto que los avances tecnológicos tienen en el sector del patrimonio. Uno de los objetivos ha sido establecer las principales líneas de usos de las TIC en el patrimonio cultural. En el amplio conjunto de TIC que presenta, es relevante el papel que adquiere el sector de las tecnologías móviles, denominado universo app y patrimonio móvil, cuyo objetivo es poner en valor los bienes patrimoniales en equipamientos patrimoniales. Los dispositivos móviles permiten presentar los contenidos al público, con informaciones multimedia y un mayor grado de individualización y personalización de las visitas, poniendo en valor el patrimonio. La sociedad de la información en la que vivimos y con las nuevas tecnologías facilitando el acceso a la información hace que esté cambiando la manera de interactuar con ella, esto es la irrupción de la realidad virtual y aumentada. Estas apps permiten experimentar con los objetivos patrimoniales gracias a las tecnologias virtuales. Las apps cuyos contenidos ofrecen reconstrucciones virtuales o realidad aumentada es una tendencia creciente.

Las TIC son actualmente una herramienta vital para la difusión del patrimonio cultural ya que han experimentado un fuerte proceso de diversificación en los últimos años y, junto a los tradicionales canales web, se han desarrollado multitud de herramientas de la web 2.0 y otros aspectos como la realidad aumentada vitales para su difusión (Timothy, 2011, en Caro et al., 2014).

\section{1. Apps y patrimonio cultural}

Es importante destacar que el aumento de las posibilidades de los smartphones, en los que se puede instalar gran cantidad de aplicaciones, amplian las funciones de los teléfonos móviles ofreciendo servicios como la búsqueda de información especializada y el uso de las redes sociales. Es interesante conocer sus principales usos en el campo del patrimonio y de la educación patrimonial.

El uso del Smartphone, especialmente las apps, está influyendo y cambiado la experiencia y el comportamiento de los visitantes. El crecimiento masivo del acceso online a través de las apps es la segunda tendencia más destacada de los próximos años (Imbert et al., 2013). En esta línea, el Anuario ACE (2017) indica cómo las apps permiten vivir una experiencia diferente y mucho más vivida en relación a la interpretación de los objetos patrimoniales. Las apps cuyos contenidos nos ofrecen reconstrucciones virtuales o realidad aumentada son las más demandadas actualmente en el sector, siendo una tendencia creciente que aventura un largo recorrido. En el campo del patrimonio, las clasificaciones de las apps se realizan según el tipo de tecnología (Ibáñez-Etxeberria et al., 2014) o según la finalidad del uso de las TIC (Anuario ACE, 2017).

Grevtsova (2016) realiza una categorización de guías móviles según criterios de clasificación del tipo de itinerario y el grado de interactividad y participación del usuario en la experiencia: guías móviles de carácter informativo, guías móviles de carácter lúdico y guías móviles de carácter colaborativo. Esta clasificación está basada en los verbos informar, jugar y construir. Las guías de carácter informativo son las más habituales en el mercado actual y su principal función es informar al usuario sobre puntos de interés de la ciudad y planificar su recorrido 
paso a paso. Hay guías informativas turísticas y apps especializadas en fomentar el conocimiento del patrimonio. En esta clasificación es donde tiene cabida la app de Fuendetodos objeto de estudio en esta investigación, que incluye un menú con los puntos más destacados de la localidad de Goya, eligiendo el visitante las opciones que más se ajustan a sus necesidades e intereses. Por su parte, las apps de carácter lúdico permiten crear actividades lúdicas y gamificadas. Los usuarios son participantes de un juego en el que deben solucionar unas tareas, resolver preguntas, etc. Las guías que permiten que el usuario se convierta en el artista y protagonista del diseño de sus propias rutas son guías de carácter colaborativo. Permiten crear contenidos personalizados, usando distintas redes de colaboración y acceder a las redes sociales, donde los usuarios comparten materiales multimedia y su opinión sobre los lugares que han visitado.

En el análisis de las aplicaciones de temática histórico-patrimonial se dan una serie de denominadores comunes (Santacana, Asensio, López, \& Martínez, 2018) que constituyen las características comunes de las aplicaciones de carácter cultural: el enfoque dominante es el turismo cultural; el recurso más importante y eficaz son las imágenes; el discurso sobre el pasado histórico o el patrimonio artístico tiene un formato descriptivo; lo anecdótico es un componente muy importante del discurso; el discurso suele ser siempre muy generalista, excepto en la anécdota; hay en el discurso una total ausencia de elementos metodológicos; en la mayoría de las aplicaciones el relato es impersonal y suele ser en tercera persona; aun cuando el discurso se refiera al pasado, es muy frecuente la ausencia de toda referencia a las fuentes; las aplicaciones referidas a las artes visuales no suelen emplear métodos de comparación con otras obras similares; existe un predominio de discursos meramente informativos, dedicando poco espacio a interacciones verdaderas y reales.

Desde el punto de vista educativo, los smartphones son herramientas educativas y las apps pueden ser objeto de aprendizaje. Las apps han de estar al servicio de la educación. El aprendizaje con dispositivos móviles mejora notablemente la comprensión de contenidos trabajados (Vicent, 2013:465-481, en Santacana et al., 2018), y los alumnos dejan de ser receptores pasivos de información y pasan a convertirse en protagonistas del proceso de aprendizaje (Anuario ACE, 2018). En Kortabitarte et al., (2017) encontramos la aplicación de la educación patrimonial a contextos educativos formales, mientras que la oferta de apps de contenido patrimonial se encuentra en constante expansión en contextos de educación informal e infrautilizan sus posibilidades educativas al no tener el usuario un papel activo (Gillate et al., 2017), a lo que se añade la dificultad de diseñar un modelo de evaluación de las apps de patrimonio cultural (Santacana et al., 2018).

\section{2. Realidad aumentada}

La tecnología de realidad aumentada (AR, de sus siglas en inglés, Augmented Reality) es un tipo de entorno virtual interactivo para enriquecer la experiencia del usuario. Consiste en insertar gráficos virtuales en nuestro espacio físico real, de modo que lo real y lo virtual consigan mezclarse ofreciendo una imagen enriquecida o aumentada de la realidad (Anuario ACE, 2017). Desde que en 1992 se acuñara el término Realidad Aumentada (Caudell, 1992), han sido numerosos los trabajos sobre la materia (Bimber, 2005; Haller, 2007; Hainich, 2010, en Torres, 2011). Esta tecnología se caracteriza por mezclar el mundo real con el virtual y se encuentra dentro del campo del patrimonio, que en combinación con los dispositivos móviles para los cuales se han diseñado algunas aplicaciones de realidad aumentada guían y enriquecen al usuario. Incluso la realidad aumentada está facilitando la accesibilidad de personas con discapacidad a espectáculos audiovisuales o que los diálogos en forma de 
subtítulos sobre la pantalla de los dispositivos de realidad aumentada puedan ser disfrutados por personas con discapacidad auditiva o sordos (Anuario ACE, 2018).

La Realidad Aumentada ofrece la posibilidad de ofrecer nuevos contenidos y ofrece interactuar con contenidos virtuales como si se tratara de objetos reales. la Realidad Aumentada muestra un gran potencial en su capacidad de mejorar el espacio real que nos circunda mediante la inclusión de gráficos generados por ordenador obteniendo una imagen mejorada o enriquecida de la realidad conformando lo que se denomina "espacios aumentados" (Ruiz Torres, 2013). El carácter educativo de este tipo de aplicaciones queda de manifiesto y supone la posibilidad de visitar el lugar del que proceden. Los recursos requeridos para ofrecer realidad aumentada son como gafas, diversos tipos de sensores con capacidad para reconocer el movimiento, sistemas de posicionamiento, etc.

La realidad aumentada nació muy vinculada a la realidad virtual. Sin embargo, es una tecnología con entidad propia. La gran diferencia con la realidad virtual es que ésta supone una inmersión completa en un mundo digital, ya sea basado en un modelo real o en uno construido, a diferencia de la realidad aumentada que fusiona el mundo real con elementos virtuales.

Para contextualizar la RA, podemos decir que nos encontramos ante una tecnología que permite la combinación de información digital e información física en tiempo real, por medio de distintos soportes tecnológicos, como por ejemplo las tablets o smartphones, y que permite crear con ello una nueva realidad (Fundación Telefónica, 2011; García et al., 2010; Muñoz, 2013; Morales, Benítez, Silva, Altamira y Mendoza, 2015; Fombona y Vázquez, 2017, en Cabero y Barroso, 2018).

Las aplicaciones que ofrece la tecnología de Realidad Aumentada para el patrimonio cultural han gozado de un gran desarrollo en los últimos años. Por una parte, debido a su gran atractivo para el público en general y, por otra, ligado a la evolución tecnológica que está sufriendo el teléfono móvil, que han hecho posible que en un solo dispositivo se pueda aplicar un sistema basado en esta tecnología mediante la introducción de un software determinado. Los sistemas de realidad aumentada combinados con geoposicionamiento y conectividad a Internet, se conforman como las aplicaciones con más futuro en lo que a difusión del patrimonio se refiere al apreciarse la realidad, es decir el recurso cultural tal como es, facilitar información antes del consumo del recurso y complementar información en el propio destino (Caro et al., 2014), configura un nuevo lugar y una oportunidad para los creadores culturales (Anuario ACE, 2018), permitiendo el desarrollo de video-guias, dispositivos ópticos inteligentes y experiencias personalizadas (Caro et al., 2015) y revelándose también como una eficaz y relevante herramienta de comunicación para dar a conocer los recursos turísticos (Fernández y Cuadrado, 2014). Sistemas de realidad aumentada o geolocalización son algunos de los ejemplos que a través de las apps han de conseguir mejorar la experiencia real del visitanteturista (Imbert et al., 2013), aportando valores añadidos a la experiencia de los usuarios (Kortabitarte et al., 2017), una construcción emergente de una educación personalizada (Olivencia \& Martínez, 2015) y cumplir una labor didáctica (Ruiz, 2014).

\section{METODOLOGÍA}

La hipótesis de partida de esta investigación se centra en el supuesto de que la utilización de las apps para móviles, y de forma concreta una app diseñada para una visita guiada por el pueblo de Fuendetodos, permite al usuario llegar a un conocimiento más completo y dinámico de la localidad y de la figura de Francisco de Goya y Lucientes. Para verificar esta hipótesis de 
trabajo, el principal objetivo de esta investigación se ha centrado en valorar cómo varía el conocimiento que los usuarios de la aplicación tienen de Goya y evaluar cómo varía su percepción sobre los valores de la localidad tras la realización de las dos rutas interpretadas por la app. La primera ruta, denominada en la app visita cultural, está centrada en la cultura y recorre la casa natal de Goya, la sala de exposiciones Ignacio Zuloaga, la iglesia, el Museo del Grabado y el taller de grabado, mientras que la otra ruta, denominada Fuendeverde, guia al visitante por lugares de la localidad de Fuendetodos como los restos del castillo exterior, el lavadero o el espacio de sensibilización ambiental Fuendeverde.

Con el fin de alcanzar los objetivos propuestos, se ha propuesto una metodología descriptiva. El análisis exhaustivo de la aplicación turística de Fuendetodos se realiza a través de una herramienta diseñada para el estudio y análisis de aplicaciones relacionadas con el patrimonio, estructurada en cinco dimensiones, que son aspectos descriptivos, informativa-comunicativa, aspectos técnicos, procesos de enseñanza-aprendizaje y dimensión patrimonial (Kortabitarte et al., 2017).

Kortabitarte et al., (2017) agrupa los distintos campos en las siguientes dimensiones: respecto a la primera, metadatos, se tiene en cuenta los agentes, el tipo de app según su funcionalidad, el precio, el idioma, la etiqueta, el año de desarrollo y la última actualización realizada. La tipología hace referencia a la app y si ésta es un itinerario de un equipamiento cultural, si es un autoitinerario con diferentes opciones para el usuario, si es un itinerario local de una ruta o más, si ofrece itinerarios nivel regional; si ofrece información puntual, si es un juego y si contiene cuentos. Idioma, uso offline, precio y accesibilidad, facilidad de acceso a la aplicación, son otros aspectos.

La vertiente informativa-comunicativa incluye aspectos tales como su potencial educomunicativo, planteando tres posibles actos comunicativos que hacen énfasis en los contenidos, en los resultados y en la reflexión. La semiótica, la interacción, las características de la información, el cifrado y la participación del usuario desde la perspectiva de propuesta por Charlene Li de poder compartir, observar, comentar, crear y curar información; y desde la perspectiva de Patterson, Kirk y Wallace sobre el tratamiento del contenido (informar, recoger, debatir y generar información).

La usabilidad, la accesibilidad técnica y el entorno seguro de la app constituyen la tercera dimensión. En cuarto lugar, los procesos de enseñanza-aprendizaje analizan los objetivos, el engagement entendido como la implicación que influye en la motivación para seguir usando la aplicación, el diseño educativo, el nivel de conocimiento según la propuesta de Webb (1997) y la potencialidad que muestra para el autoaprendizaje. También se tiene en cuenta el tipo de conocimiento que genera, conceptual, procedimental y actitudinal, o una mezcla de ellos.

Finalmente, en la dimensión patrimonial, se tiene en cuenta la tipología y la narrativa patrimonial. La variable tipología patrimonial tiene por objetivo conocer qué tipologias patrimoniales son las más trabajadas por las apps (Aierdi et al., 2017). Se analiza la concepción que los agentes que lanzan las apps tienen para relacionarlas con los contenidos y los objetivos implícitos de las apps. Indicadores de la sensibilización hacia la cadena patrimonial, la concienciación y el esfuerzo para la proyección social y su vinculación a la Educación Patrimonial son otros criterios de análisis.

\section{RESULTADOS Y DISCUSIÓN}

Fuendetodos es una aplicación turística para móviles con información sobre el municipio e interacciones de realidad aumentada que permiten sacarse una foto junto a un retrato de 
Francisco de Goya en la casa natal del pintor o que los niños vean en tres dimensiones uno de los asnos que aparecen en la serie de Los Caprichos de Goya (DPZ, 2018).

Los datos obtenidos del análisis de la app pueden concretarse en los siguientes aspectos:

En primer lugar, la dimensión descriptiva, puesto que se trata de una aplicación desarrollada por la empresa Imascono Art S.L. y cuenta con el apoyo económico del Consorcio GoyaFuendetodos y está disponible para Android e IOS, aunque sólo puede activarse una vez que el visitante está en la localidad de Fuendetodos. Se trata de una app gratuita y ocupa 99,7 MB y va dirigida a todo tipo de públicos en general, aunque también hay una parte más lúdica e infantil. Está disponible en castellano, la etiqueta viajes es la que se ha añadido en la store para su descarga.

Figura 1. Pantallas de la aplicación para móviles Fuendetodos
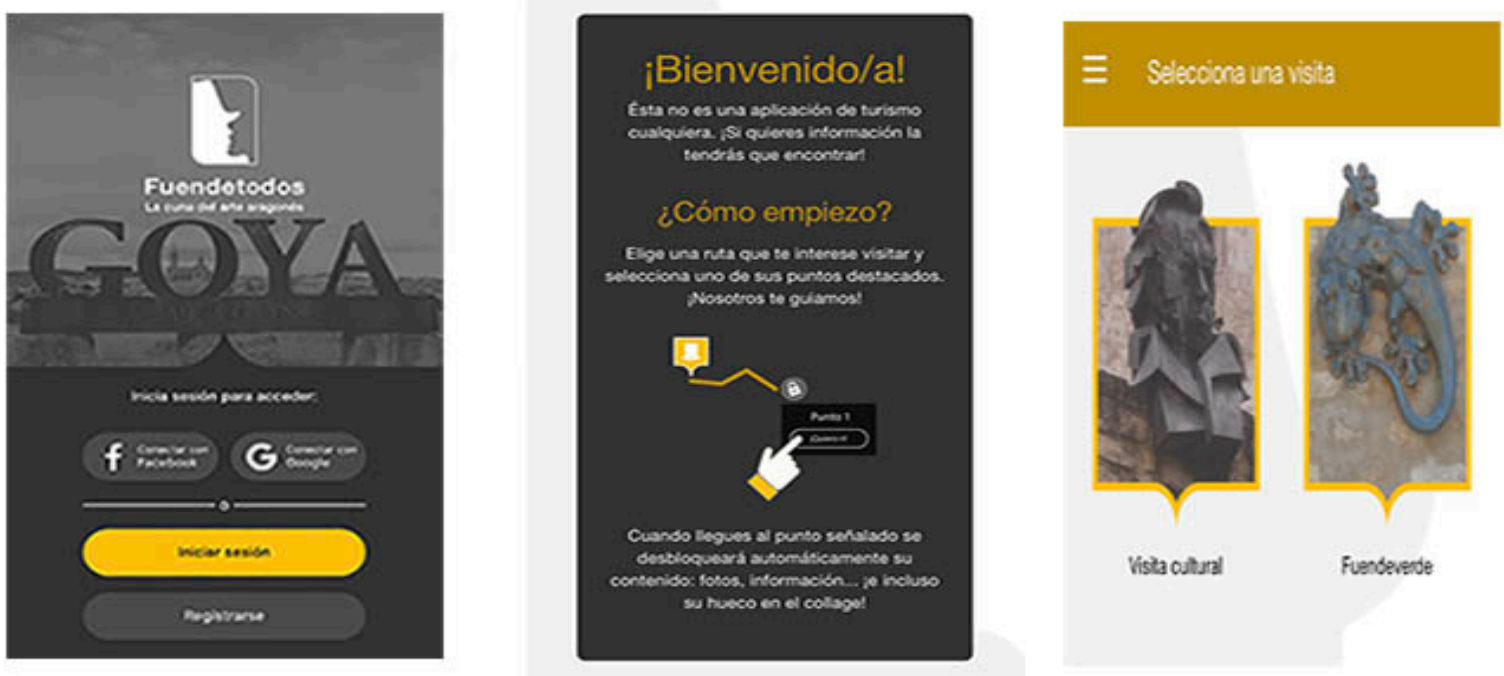

Fuente: Imascono Art S.L.

Dentro de la dimensión comunicativa, la app está creada con un fin didáctico, con especial hincapié en los contenidos y una mera transmisión de información. Todo ello para que el visitante pueda aprender a través de la visita cultural cómo era el Museo del grabado, con información sobre la creación de este museo y las cuatro series de grabados de Goya que se pueden visitar: Disparates, Tauromaquia, Caprichos y Desastres de la guerra; la Casa natal, donde transcurrieron los seis primeros años de la infancia de Francisco de Goya y que sigue conservando el aspecto que tuvo en la época; el Taller de grabado profesional; la iglesia y escultura de Goya; la Sala Zuloaga y conocer el espacio Fuendeverde y el entorno, con actividades para los visitantes. En definitiva, la app trata de dar a conocer y ofrecer información sobre los distintos lugares que forman parte del patrimonio de la localidad zaragozana. En cuanto a la intencionalidad discursiva, en la app de Fuendetodos se incide en los contenidos. Además, hay espacio para el juego pudiendo el usuario realizar fotos que se añaden al collage al final de la visita y que puede ser compartido por redes sociales o email.

Los recursos más utilizados para la creación de contenidos son el texto, imágenes y mapas. Cada espacio de la visita cultural es descrito mediante un texto informativo sencillo y eficaz que ayuda al visitante a conocer detalles específicos del lugar en concreto y se acompaña de 
imágenes ilustrativas, así como mapas para llegar a cada uno de los lugares propuestos en la visita cultural.

Relacionado con el campo del diseño educativo, la variable sobre los recursos para profundizar en la temática e información de las apps, esta aplicación no está dotada de recursos que ayuden al usuario a profundizar en los conceptos e informaciones que quiere transmitir la app. La posibilidad de crear contenidos a través de la app no es posible y el usuario solamente se informa de los lugares de interés ofrecidos por la app, resultando una comunicación unilateral.

La variable tipología utilizada es el itinerario donde el usuario es guiado mediante el recorrido que muestra la app. De este modo, el usuario puede visitar los distintos lugares de interés gracias a los mapas propuestos. El usuario solo es observador y no se produce un aprendizaje activo.

Figura 2. Pantallas de la aplicación para móviles Fuendetodos
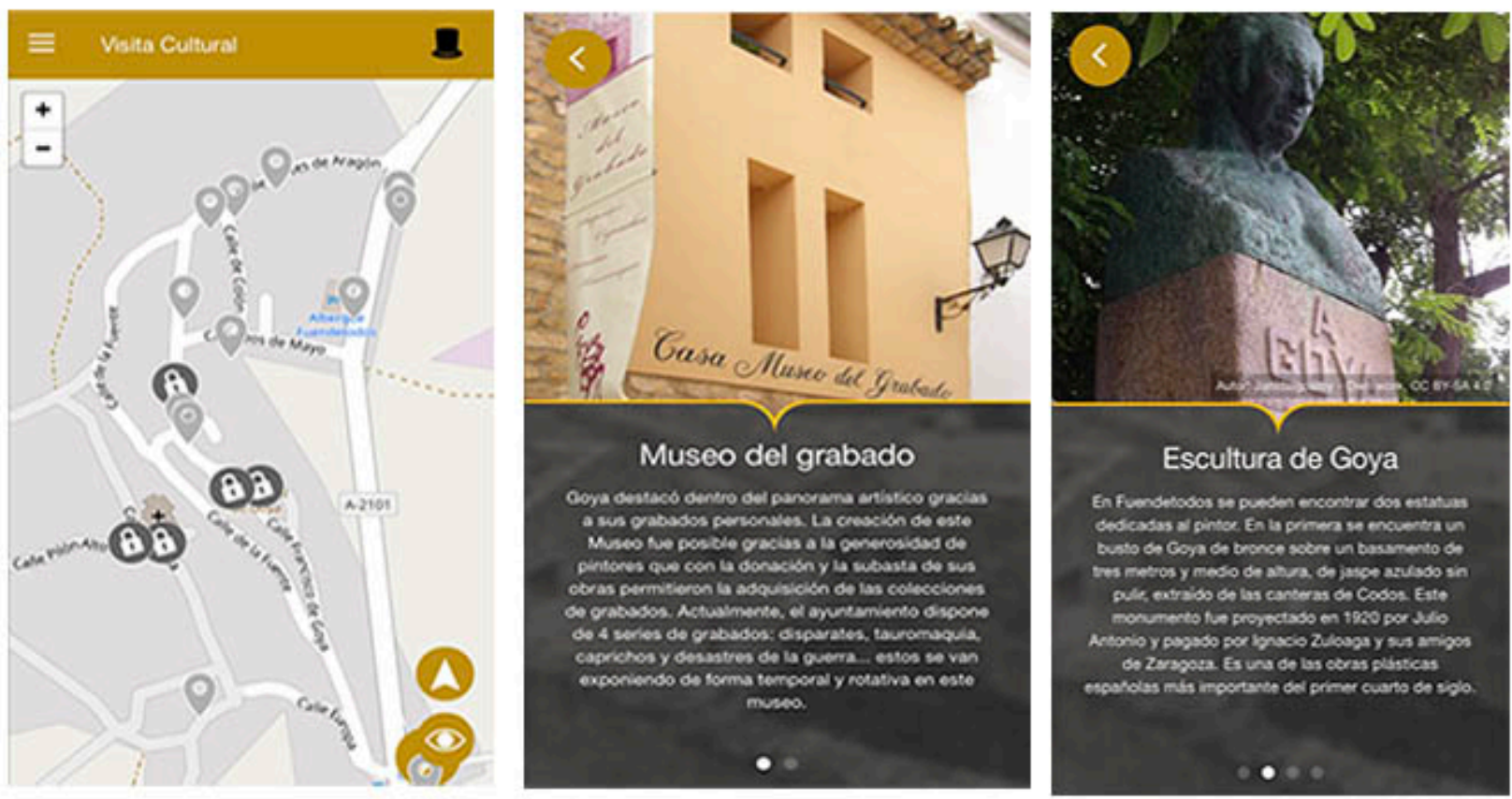

Fuente: Imascono Art S.L.

La app está presente en las distintas fases del proceso de enseñanza-aprendizaje de los lugares de interés: antes de la visita ofrece información práctica y conocer de antemano qué pueden encontrar los visitantes en cada uno de los edificios de interés; durante la visita, con elementos de engagement que permiten enriquecer la experiencia cuando se está visitando el lugar elegido de interés; y tras la realización de la visita, donde la app permite al usuario guardar las fotos hechas.

Se utiliza la realidad aumentada, permitiendo la visualización de información mezclada con objetos reales, en el interior de la casa natal de Goya, pudiendo el visitante conocer el estudio que tenía en el desván, pudiendo también posar ante la cámara para guardar un recuerdo de la visita. Caro et al. (2015) señalan que los sistemas de realidad aumentada sean quizás las aplicaciones con más futuro dentro de la difusión del patrimonio cultural ya que permiten que se aprecie la realidad, el recurso cultural tal como es, puede completar la información antes de 
llegada del turista al recurso, y complementar la información en el propio destino, aumentando la información visual en la propia visita al bien de interés cultural, y la tecnología de realidad aumentada incorpora un valor añadido a la experiencia del turista gracias a los dispositivos móviles.

Figura 3. Pantallas de la aplicación para móviles Fuendetodos
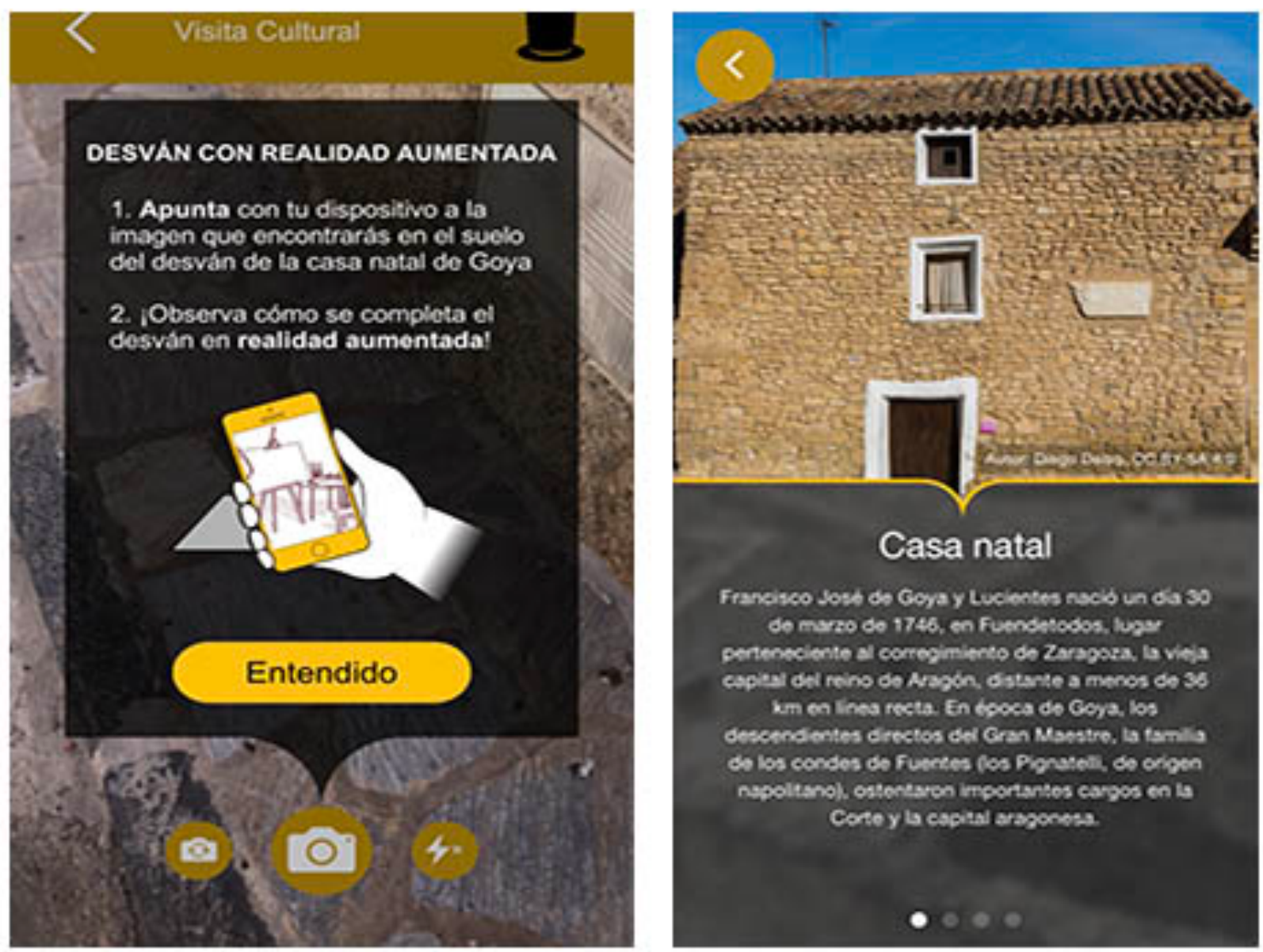

Fuente: Imascono Art S.L.

Si atendemos a la dimensión técnica, se recogen aspectos como la usabilidad, la accesibilidad técnica y el entorno seguro. La aplicación es para todos los públicos, y también dispone de alguna funcionalidad para niños, permitiendo que vean en tres dimensiones uno de los asnos de la serie de Los Caprichos de Goya. Está sobre todo enfocada a un público joven y familiarizado con el uso de las nuevas tecnologías. Es de fácil uso, manejable y accesible para todos. El usuario no puede personalizar la app, y solamente puede realizar una serie de ajustes, referentes al idioma, la posibilidad de notificaciones, politica de privacidad e información sobre la Fundación Fuendetodos. Una de las limitaciones de la app es que requiere estar in situ para su funcionamiento. Solo pueden tener acceso al contenido los usuarios que visiten la localidad de Fuendetodos. No se aprovecha la potencialidad de las apps que si permiten crear contenidos que permitirian el enriquecimiento de la información facilitada por la app analizada. 
Figura 4. Pantallas de la aplicación para móviles Fuendetodos
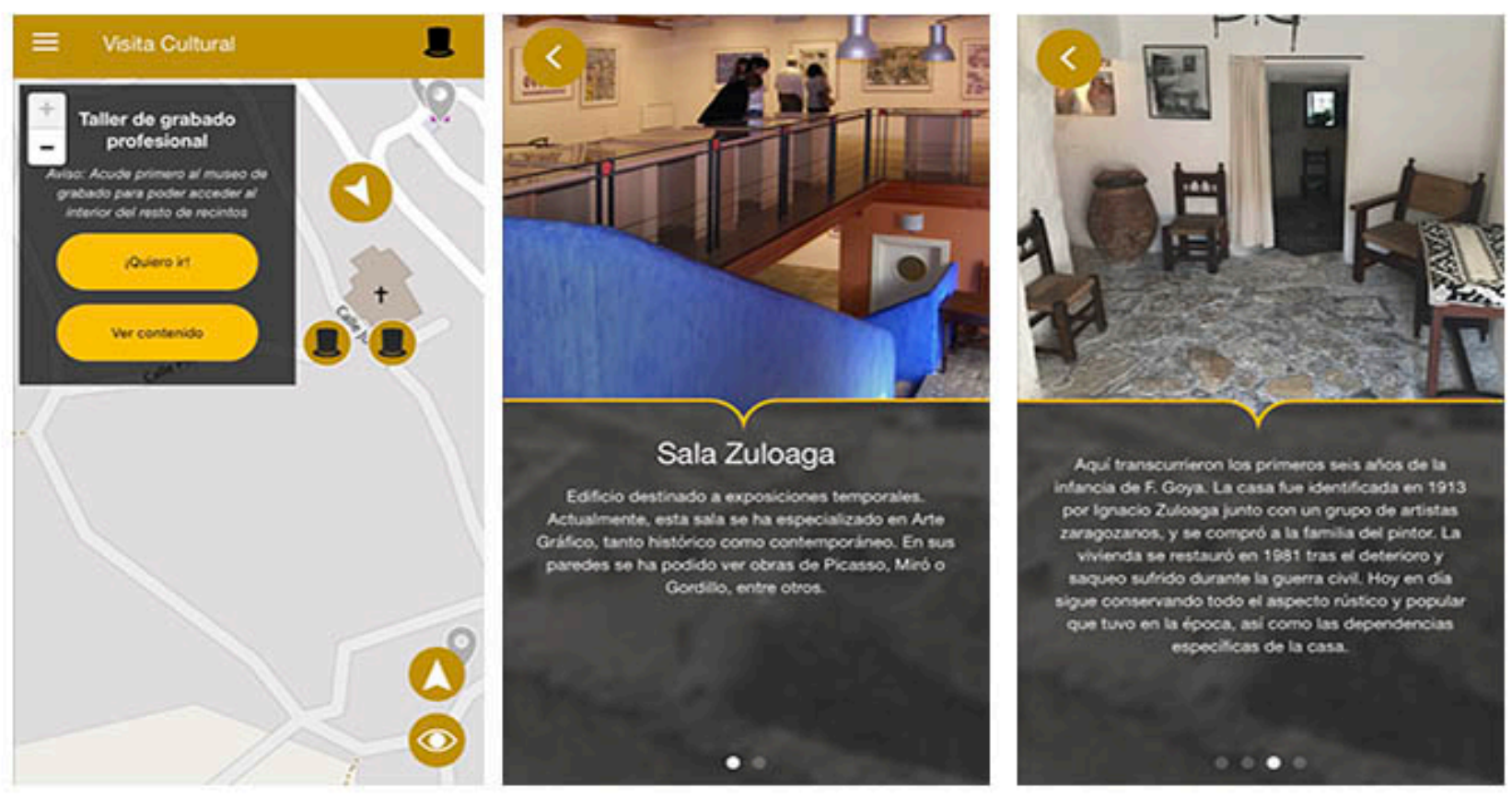

Fuente: Imascono Art S.L.

La cuarta dimensión de análisis se refiere a los procesos de enseñanza-aprendizaje. El engagement de esta app viene dada por la opción de compartir el collage creado por el usuario por email y redes sociales. La app es un proyecto de gran envergadura que ayuda a la valorización del patrimonio de la localidad, basada en un itinerario guiado organizado por espacio físico y donde la interacción social se limita a que los usuarios puedan compartir contenidos por las redes sociales o por email. En cuanto a las acciones específicas e intencionalidad de la app, se permite que el usuario no tenga un nivel de conocimiento previo ni habilidades digitales específicas para su manejoa. Responde a un modelo de itinerario centrado en los contenidos que ofrece información, basado en la transmisión de información y con interactividad para favorecer el engagement, ofreciendo recursos como la realidad aumentada y elementos de gamificación,

Resulta significativo la visión que se transmite sobre la concepción del patrimonio en la app, con una perspectiva integradora de carácter histórico, con referentes documentales, permitiendo el conocimiento de los principales lugares de interés que se pueden visitar.

Si bien en este estudio la app de Fuendetodos aplicada al patrimonio es un agente de difusión de la localidad y de sus principales puntos de interés así como de la figura de Goya a través del recorrido por los lugares más importantes para el pintor, la posibilidad de interactuar, crear o modificar el contenido convertiría al usuario en protagonista, pero este aspecto no está representado en esta aplicación, por lo que la potencialidad de la app está limitada a la transmisión unilateral de contenido e incitando poco a la participación. 


\section{CONCLUSIONES}

En este trabajo se ha planteado la hipótesis de si la utilización de la app Fuendetodos permitiría al usuario llegar a generar un conocimiento más completo y dinámico de la figura y presencia de Goya en la localidad de Fuendetodos. Esta hipótesis se ha verificado positivamente. El análisis de los aspectos descriptivos, comunicativos, técnico, de enseñanzaaprendizaje y patrimonial de la app ha permitido determinar que los usuarios de la aplicación pueden incrementar el conocimiento que tenían de Francisco de Goya y su presencia en Fuendetodos. Además, tras la realización de los dos itinerarios propuestos por la app, una visita cultural y la ruta Fuendeverde, la percepción del visitante puede variar gracias a la información y la experiencia vivida. Los usuarios tendrán una opinión más formada y rigurosa de la obra de Goya. Las nuevas tecnologías, y en este caso, una aplicación para móvil, contribuyen a completar la opinión que los usuarios tenían del pintor.

La utilización de la aplicación genera un aprendizaje y permite a los usuarios ser partícipes de dicho proceso. Las propuestas de educación patrimonial ofertadas desde los espacios de educación informal (museos, sitios patrimoniales, ciudades, etc.) se han diversificado y la oferta de apps para este campo parece ser cada vez mayor (Guillate, 2017). Fuendetodos se enmarca en un contexto de enseñanza de aprendizaje informal. La app favorece una experiencia cercana, divertida y experiencial para el visitante de la localidad y centra su atención en dos itinerarios principales perfectamente delimitados. Grevtsova (2016) y Kortabitarte el al. (2018) señalan que la mayoría de las apps en torno al patrimonio se dedican a informar y están diseñadas para un perfil de turista.

La app Fuendetodos se concibe como una aplicación dirigida al turismo, con un aprendizaje informal y donde los usuarios juegan un papel pasivo. Las apps serian herramientas de apoyo facilitadoras de experiencias educativas, pero su buen aprovechamiento dependerá de su diseño, que a su vez dependerá de los objetivos que se planteen los gestores patrimoniales (Guillate, 2017).

El análisis arroja que la app se centra en los contenidos, primando un modelo educomunicativo centrado en ofrecer contenidos sobre los sitios de interés elaborados previamente, y una pequeña oferta de participación activa de los usuarios que se reduce a la posibilidad de compartir un collage en las redes sociales o utilizar la realidad aumentada. Es una app basada en dar información sobre el patrimonio histórico y artístico de la localidad.

La aplicación es para todos los públicos, y también dispone de alguna funcionalidad para niños, permitiendo que vean en tres dimensiones uno de los asnos de la serie de Los Caprichos de Goya. Está sobre todo enfocada a un público joven y familiarizado con el uso de las nuevas tecnologías.

En la app, la realidad aumentada es una de las principales atracciones. Los usuarios pueden hacerse fotografias y compartirlas por redes sociales. Esta propuesta de realidad aumentada enriquece de contenido a los lugares históricos al añadir elementos de interés y mostrar al visitante de forma atractiva, lúdica y didáctica unos contenidos. La realidad aumentada es un valor añadido para la experiencia de los usuarios. La introducción de la geolocalización, la realidad aumentada y la realidad virtual pueden aportar valores añadidos a la experiencia de los usuarios, además de la gamificación y la incorporación de las redes sociales, que posibilitan un tipo de interacción que las apps de un estilo más tradicional no ofrecían (Guillate, 2017). La realidad aumentada es una eficaz y relevante herramienta de comunicación a través de la cual es posible dar a conocer los recursos turísticos, de ahí que su uso en este sector y, particularmente, su aplicación al turismo cultural, se haya convertido en un valor añadido 
para dar a conocer el patrimonio histórico y cultural de un destino turístico (Alles y Marqués, 2014).

Una de las limitaciones de la app de Fuendetodos es que requiere estar in situ para su funcionamiento. Solo pueden tener acceso al contenido aquellos usuarios que visiten la localidad de Fuendetodos, por tanto su utilización en otros contextos educativos formales no sería posible. En este aspecto, para facilitar su uso en otros contextos formales, sería interesante poder facilitar recursos como plantillas descargables o códigos que permitan utilizar estas apps dentro del aula y sin encontrarse en el espacio patrimonial (Kortabitarte et al., 2018).

Desde una perspectiva más amplia, la posibilidad de disponer la localidad de Fuendetodos de una aplicación turística permite generar valor agregado a las experiencias de los turistas. Estas aplicaciones favorecen en el entorno turístico la promoción de destinos, servicios y del patrimonio histórico y a la vez ofrecen a los turistas experiencias personalizadas. Las TIC son actualmente una herramienta de especial interés para la difusión del patrimonio cultural ya que han experimentado un fuerte proceso de diversificación en los últimos años y, junto a los tradicionales canales web, se han desarrollado multitud de herramientas de la web 2.0 y otros aspectos como la realidad aumentada vitales para su difusión (Timothy, 2011, en Caro et al., 2015). Retos y oportunidades que ofrece la tecnología que los destinos turísticos deben aprovechar para darse a conocer, el desarrollo y la promoción del territorio, sobre todo en municipios rurales como Fuendetodos que se enfrentan al problema de la despoblación.

\section{BIBLIOGRAFÍA}

Akcayir, Murat y Akcayir Gökce (2017): "Advantages and challenges associated with augmented reality for education: A systematic review of the literatura”, Educational Research Review, 20:1-11.

Cabero, Julio y Barroso, Julio Manuel (2016): "Posibilidades educativas de la realidad aumentada", Journal of New Approaches in Educational Research, 5 (1): 46-52.

Caro, José Luis, Luque, Ana y Zayas, Belén (2015): "Nuevas tecnologías para la interpretación y promoción de los recursos turísticos culturales", Pasos, Revista de Turismo y Patrimonio Cultural, 13(4).

Cuenca, José María (2003): "Análisis de concepciones sobre la enseñanza del patrimonio en la educación obligatoria”, Enseñanza de las Ciencias Sociales, 2: 37-45.

Diputación Provincial de Zaragoza, DPZ (2018): http://www.dpz.es/noticias/fuendetodos-lanza-una-aplicacion-turistica-para-moviles-coninformacion-sobre-el-municipio-e-interacciones-de-realidad-aumentada

Etxeberria, Alex, Vicent, Naiara, Asensio, Mikel, Cuenca, José María y Fontal, Olaia (2014): "Learning in archaeological sites with mobile devices", Munibe Antropologia-Arkeologia, 65: 313-321.

Fernández, María Teresa y Cuadrado, Ramón (2014): "El impacto de las nuevas tecnologías en el sector turístico: Aplicación de la realidad aumentada al turismo cultural", International Journal of World of Tourism, 1(2).

Fombona Javier, Pascual, María Ángeles, Madeira, María Filomena (2012): "Realidad aumentada, una evolución de las aplicaciones de los dispositivos móviles", Pixel-Bit. Revista de Medios y Educación, 2012, 41: 197-210. 
García-Prieto, Ana María y González, Rocío (2015): "Análisis de la aplicación de la realidad aumentada en el sector turístico: Una propuesta de mejora”, Gran Tour, 10: 52-72.

Gillate, Iratxe, Vicent, Naiara, Gómez, Carmen y Marín-Cepeda, Sofia (2017): "Características y dimensión educativa en apps de educación patrimonial: Análisis a partir del método OEPE”, Estudios Pedagógicos (Valdivia), 43(4): 115-136.

Grevtsova, Irina (2013): "El patrimonio urbano al alcance de la mano: Arquitectura, urbanismo y apps", Her\&Mus. Heritage \& Museography, 13: 36-43.

Grevtsova, Irina y Grupo Dhigecs (2016). “Tendencias del uso de las tecnologías móviles en espacios urbanos: m-learning y patrimonio cultural”, Revista PH, 90: 132-151.

Ibáñez-Etxeberria, Alex, Fontal, Olaia y Rivero, Pilar (2018): "Educación patrimonial y TIC en España: Marco normativo, variables estructurantes y programas referentes", Arbor, 194, 788: 448.

Ibáñez-Etxeberria, Alex, Kortabitarte, Aroia, De Castro, Pablo y Gillate, Iratxe (2019): "Competencia digital mediante apps de temática patrimonial en el marco DigComp", Revista Electrónica Interuniversitaria De Formación Del Profesorado, 22(1):13-27.

Imbert-Bouchard, Daniel, Llonch, Nayra, Martín, Carolina y Osàcar, Eugeni (2013): "Turismo cultural y APPs. Un breve panorama de la situación actual", Her\&Mus. Heritage \& Museography, 13: 44-54.

Ibáñez-Etxeberria, Alex y Kortabitarte Aroia, (2016): “Apps, redes sociales y dispositivos móviles en educacion patrimonial", Plan Nacional de Educación y patrimonio, Proyectos de Investigación.

Kortabitarte, Aroia, Ibáñez-Etxeberria, Alex, Luna, Ursula, Vicent, Naiara, Gillate, Iratxe, Molero, Begoña y Kintana, Jurgi (2017): "Dimensiones para la evaluación de aprendizajes en APPs sobre patrimonio", Pulso, 40: 17-33.

Leiva, Juan José y Moreno, Noelia (2015): “Tecnologías de geolocalización y realidad aumentada en contextos educativos: Experiencias y herramientas didácticas", DIM: Didáctica, Innovación y Multimedia, 31:1-18.

Luna, Úrsula, Ibáñez, Álex y Kortabitarte, Aroia (2019): "Herramientas digitales innovadoras en espacios educativos. El uso de las apps para la enseñanza-aprendizaje del patrimonio en España". en Juan Calvillo Barrios (coord.) Escenarios, heterogeneidad y nuevas perspectivas para la comprensión de la educación, Consorcio Educativo de Oriente, Universidad de Oriente, pp. 90-106.

Rascón, Sebastián y Sánchez, Ana Lucía (2008): "Las nuevas tecnologías aplicadas a la didáctica del patrimonio", Pulso, 31: 67-92.

Martínez, Tania, López, Victoria, Asensio, Mikel y Santacana, Joan (2018): “¿Entretener o aprender? la evaluación de las tecnologías móviles en la didáctica del patrimonio", Ensayos: Revista de la Facultad de Educación de Albacete, 33(1): 39-52.

Morère, Nuria, Perelló, Salvador, Dell’Orto, Valentina y Martín, Clara (2013): Turismo cultural: Patrimonio, museos y empleabilidad, EOI Escuela de Organización Industrial.

Prendes, Carlos (2015): "Realidad aumentada y educación: Análisis de experiencias prácticas”, Píxel-Bit.Revista De Medios y Educación, 46:187-203.

Ruiz, David (2011): "Realidad aumentada, educación y museos", Revista ICONO14 Revista Científica De Comunicación y Tecnologias Emergentes, 9(2): 212-226.

Santacana, Joan, Asensio, Mikel, López, Victoria y Martínez, Tània (2018): La evaluación de las APPs en el patrimonio cultural, Gijón, Ediciones Trea. 